\title{
A CLOSE LOOK AT NGC1068 WITH ADAPTIVE OPTICS: DUST TORUS AND MICRO-SPIRAL STRUCTURE
}

\author{
DANIEL ROUAN AND OLIVIER LAI \\ Observatoire de Paris, F92195, Meudon, France \\ DANIELLE ALLOIN \\ SAp-CEA Saclay, F91191 Gif sur Yvette, France \\ AND \\ FRANCOIS RIGAUT \\ CFHT, Kamuela, Hawaii, 96743, USA
}

\section{Introduction}

As the closest Seyfert nucleus, NGC 1068 is a key object to establish on firm grounds the model of active galactic nuclei (AGN). Two important questions relative to AGNs are : $i$ ) can we actually see the parsec-scale torus of dust and molecular gas around the central engine predicted by the popular Unified Model model (e.g. Antonucci, 1993) ?; ii) how the gas is brought down to the parsec scale to feed the monster, and, in particular, is there a micro spiral/bar system that could produce the required torque ? Adaptive Optics in the near-IR has potential advantages to make a significant progress in those two questions : the low dust opacity and the excellent angular resolution.

\section{Results}

We obtained diffraction-limited near-IR images in $\mathrm{J}, \mathrm{H}$ and $\mathrm{K}$ of the nucleus of NGC 1068, in February 97 with the Adaptive Optics system of CFHT (Rigaut et al., 1995)and the Monica near-IR camera. The achieved resolution (0.13") reveals several structures, particularly prominent on the [J-K] image shown on the Fig. $1:$ a) a belt along the ESE-WNW direction, on both side of an unresolved core, beginning at $\approx 15 \mathrm{pc}(0.18 ")$ and extending up to $120 \mathrm{pc}(1.5 ") ; \mathrm{b})$ a central S-shaped structure of diameter $\approx 40 \mathrm{pc}$. A precise positionning of the IR peak was done relative to the HST I band 


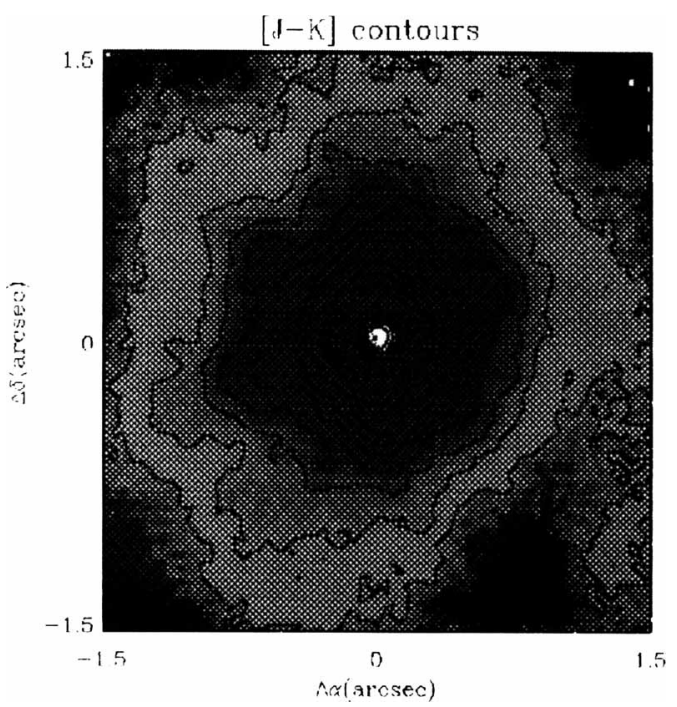

Figure 1. [J-K] image + isophotes $)$ of the $3 \times 3(\operatorname{arcsec})^{c}$ around the nucleus of NGC1068

peak, within 0.1" from the admitted location of the true AGN (radio source S1). These data are largely consistent with the Unified Model of AGN: the near-IR core is likely the emission from the hot inner (unresolved) walls of the dust/molecular torus, while the belt is most probably the trace of warm dust in the torus itself, showing up at a distance where the bright central source is no longer dazzling. This interpretation is supported by several facts: a) the belt is perpendicular to the local radio jet originating at $\mathrm{S} 1$; b) its direction follows exactly that of the disk of ionized gas recently found with the VLBA (Gallimore et al., 1997) ; c) the flux is consistent with dust at $600 \mathrm{~K}$, the temperature expected at this distance from the AGN. Regarding the $\mathrm{S}$-shaped feature, its near-IR flux is consistent with radio data, if free-free emission from ionized gas, but we rather propose that the $S$ is indeed an extremely compact (40 pc diameter) barred spiral structure, that would be the innermost of imbricated spiral structures predicted by models (Combes, 1994). This is supported by the stellar distribution - deduced from the $J$ image -, which follows precisely an exponential disk with a 20 pc scale-length : exactly the distribution resulting from a bar of this size.

\section{References}

Antonucci R., 1993, ARAA 31, 473-521

Combes F., 1994, in "The formation and Evolution of Galaxies", C. Munõz \& F. Sanchez Eds

Gallimore J.F., Baum, S.A., D'Dea C.P., 1997, preprint (web site)

Rigaut F., Arsenault R., Kerr J., et al. ,1995, SPIE Conference Proceedings, Vol. 2201 , p. 149 